\title{
Optimal positions of translational vibration absorbers in vibroisolation of mechanical press
}

\author{
Waldemar Łatas ${ }^{*}$ \\ Institute of Applied Mechanics, Cracow University of Technology, \\ Al. Jana Pawła II 37, 31-864 Kraków, Poland
}

\begin{abstract}
The paper deals with the problem of optimizing the positions of dynamic vibration absorbers in a mechanical press subjected to harmonic excitation force. Assuming flat motion of a rigid body and small vibrations, a discrete linear dynamic model of the press with absorbers is constructed. Using the results of numerical calculations, the optimal positions of the single or two vibration absorbers tuned to the excitation frequency, for different direction of excitation force, are obtained.
\end{abstract}

\section{Introduction}

Tuned mass dampers or dynamic vibration absorbers (i.e. TMDs with no damping) are simple and effective vibration suppressing devices, employed in a variety of problems of different scale and frequency range. In civil engineering TMDs are used in structures with low internal damping and low natural frequencies, such as suspension and cable-stayed bridges, high-rise buildings, masts, chimneys and wind power towers, exposed to wind or earthquake excitations [1-4]. In road and railway bridges, as well as foot bridges, where tuned mass dampers are also widely used, the excitation forces come from vehicle and pedestrian traffic [5-6]. On a smaller case TMDs are efficient devices in industry for vibration protection of equipment, e.g. for high-speed machining chatter suppression [7]. Many theoretical works were devoted to optimization of the tuned mass dampers, of passive, active or semi-active types, for different types of excitations, in both linear and non-linear problems [8-11].

During the operation of machinery such as vibrating screens, mills, textile machines, fans, or presses, occurs dynamic forces transmitted to the ground [12], causing undesirable vibrations and noise in the work area and in the surrounding environment (so called structure borne noise and vibrations). Large presses can generate vibrations of frequencies which may coincide with natural frequencies of the floors or walls. The most important issue is the machine isolation by the proper choice of elastic and damping support parameters. If the isolation is not sufficient, it is necessary to use additional means, for example passive tuned mass dampers may be helpful due to the cost and reliability issues. In such situations determining the optimal positions of dampers, which may be limited by technical consideration, is an additional task.

* Corresponding author: latas@mech.pk.edu.pl 
The paper presents the problem of vibroisolation of a mechanical press, subjected to inertial excitation due to the reciprocating motion of machine components. Such excitation commonly is of a polyharmonic type, due to the periodic movements of slides along the guides in opposite directions, resulted from the rotation of appropriately shaped cams. Vibrations of the presses in a hall are transmitted to the ground and may cause structure borne vibrations in the surroundings, for example the floors in the adjacent buildings.

If the excitation force spectrum contains significant components of very low frequencies, it may not be possible to use the supercritical vibroisolation (i.e. supports with very low stiffness), even with the pneumatic springs, due to the economical or technical reasons. Then, the used damping pads (often made of elastomers) may amplify some of the harmonics of the excitation force, resulting in exceeding the admissible levels in office rooms due to the impact of vibrations on the human body.

The aim of the paper is to determine the optimal positions and tuning of translational dynamic vibration absorbers (DVA, i.e. the TMDs with no damping device) suppressing the selected harmonics of the force transmitted to the ground. The use of the resonant devices is justified due to the stable working conditions, because drive shaft of the press always rotates at a constant angular velocity.

In numerical calculations, one absorber (horizontal or vertical) and a system of two absorbers (horizontal and vertical) are considered. Because the harmonics relevant to the accuracy of the technological process may vary from those harmonics that are important due to the impact on the human body, a wide frequency range including the all natural frequencies of the system is taken into account.

\section{Description of the system}

Figure 1 presents the diagram of press resting on four supports, excited by the resultant inertial force $F(t)$. The mass of the press equals $M=11000 \mathrm{~kg}$, its moment of inertia about the axis passing through the center of mass equals $J_{C}=4020 \mathrm{kgm}^{2}$, the geometric dimensions are: $b=0.75 \mathrm{~m}, a=0.90 \mathrm{~m}$, the angle of inclination of the force is $\beta_{0}=45^{\circ}$.

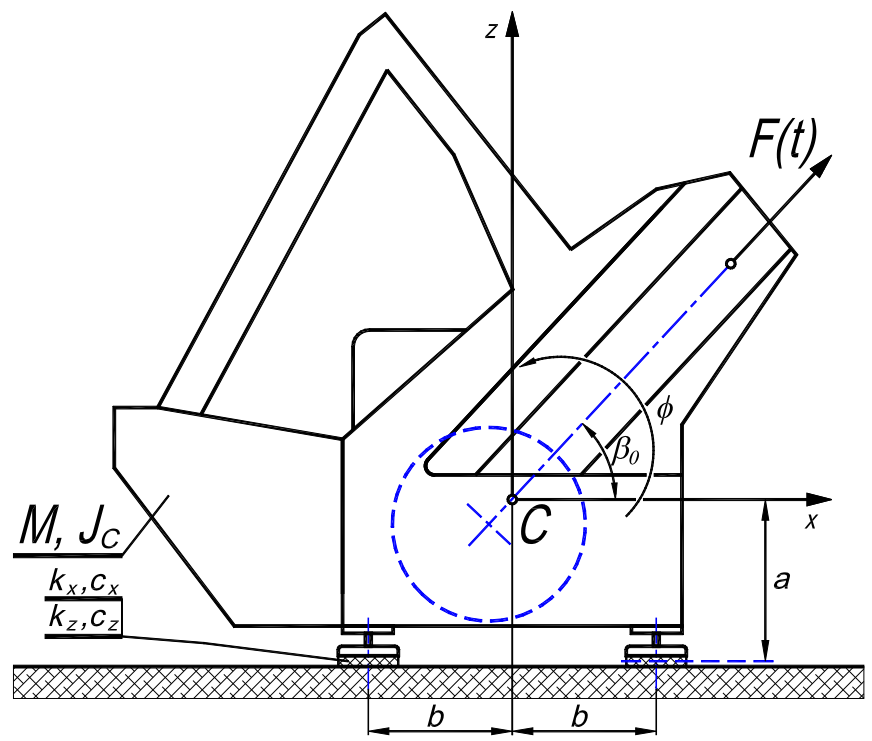

Fig. 1. Press diagram without attached tuned mass dampers. 
The horizontal and vertical stiffness and the respective damping coefficients of the supports made of the elastomer pads are as follows: $k_{x}=760 \cdot 10^{5} \mathrm{~N} / \mathrm{m}$, $c_{x}=280 \cdot 10^{3} \mathrm{Ns} / \mathrm{m}, \quad k_{z}=670 \cdot 10^{6} \mathrm{~N} / \mathrm{m}, \quad c_{z}=540 \cdot 10^{3} \mathrm{Ns} / \mathrm{m} . \quad$ The dimensionless damping coefficients in the horizontal and vertical directions are: $\zeta_{x}=0.31, \zeta_{z}=0.20$. The three natural frequencies of the press are approximately (when ignoring damping): $f_{1}=24 \mathrm{~Hz}, f_{2}=78 \mathrm{~Hz}, f_{3}=106 \mathrm{~Hz}$.

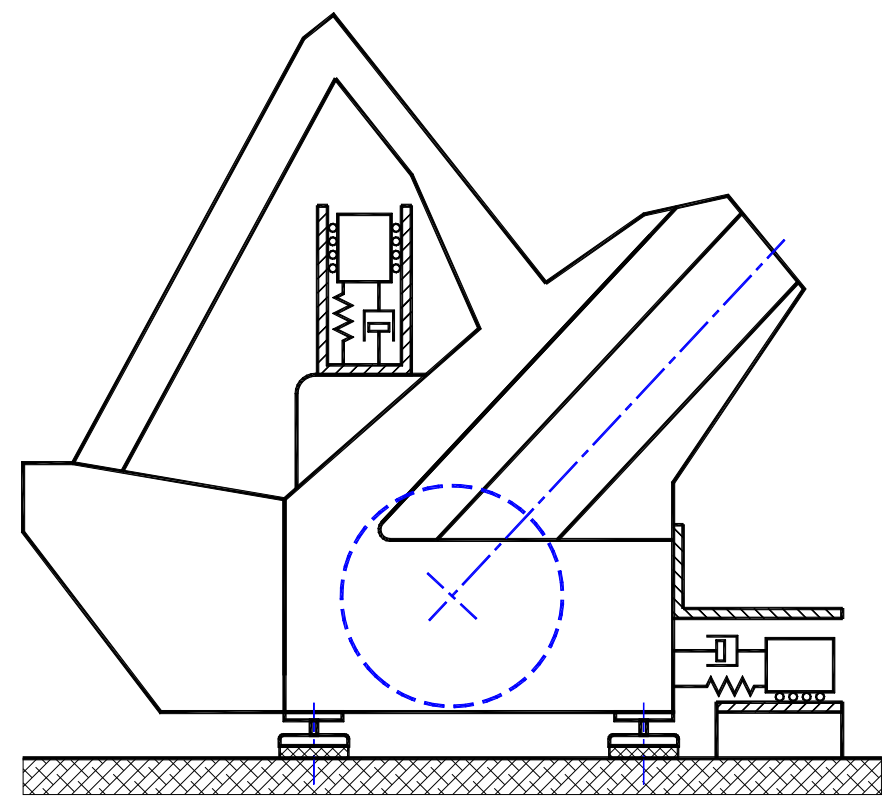

Fig. 2. Press with attached the horizontal and the vertical tuned mass dampers.

Figure 2 shows the press with two attached mass dampers, the vertical moving along the slide attached to the machine and the horizontal moving along the slide fixed to the ground. These dampers move in different ways, but with some simplifying assumptions (mainly neglecting the nonlinear terms) both can be described with the same theoretical model.

The mass dampers have a simple principle of operation, but their effectiveness, in addition to the proper selection of physical parameters, depends significantly on the appropriate location. The best location may not be obvious when the vibrations in different directions are coupled.

\section{Model of the press with attached tuned mass dampers}

The computational model presented, of a linear type, was built with the assumptions of small vibrations, what is commonly accepted in the vibroisolation problems.

Figure 3 presents a press diagram with a system of two horizontal and two vertical mass dampers attached. The number of mass dampers could be higher, but it is not justified in the considered problem, where the aim is to find the simple and sufficient dampers configuration. Relating to the differences between the dampers mountings shown in Fig. 2, it is assumed that all dampers move along the slides fixed to the ground, without investigating the problems of technical nature. 


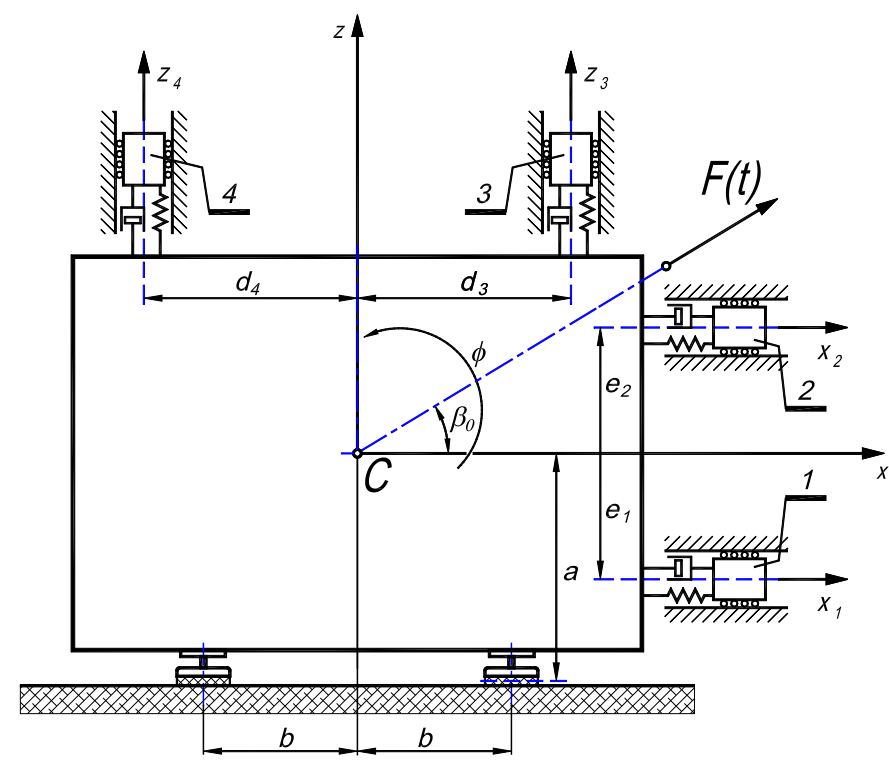

Fig. 3. Schematic arrangement of the horizontal and vertical tuned mass dampers.

In Fig. 3 the dampers are placed outside of the contour of the machine, but they can be moved to the inside of the contour along the motion directions. Additionally, in order to avoid rotating of the press with respect to the vertical axis, one damper in the diagram represents in practice two symmetrically mounted dampers (with its parameters decreased twice).

A flat motion of the system is assumed, described by the following coordinates: $x_{C}, z_{C}$ - press mass center displacements in the horizontal and vertical direction, $\varphi$ - press rotation angle; $x_{1}, x_{2}$ - displacements of the horizontal dampers; $z_{1}, z_{2}$ - displacements of the vertical dampers.

The vibrations of the press with the system of mass dampers attached (shown in Fig. 3) are described by the system of linear differential equations:

$$
\begin{aligned}
& M \ddot{x}_{C}+\left(4 c_{x}+c_{1}+c_{2}\right) \dot{x}_{C}+\left(4 c_{x} a+c_{1} e_{1}-c_{2} e_{2}\right) \dot{\varphi}-c_{1} \dot{x}_{1}-c_{2} \dot{x}_{2}+\left(4 k_{x}+k_{1}+k_{2}\right) x_{C} \\
& +\left(4 k_{x} a+k_{1} e_{1}-k_{2} e_{2}\right) \varphi-k_{1} x_{1}-k_{2} x_{2}=F(t) \cos \beta_{0} \\
& M \ddot{z}_{C}+\left(4 c_{z}+c_{3}+c_{4}\right) \dot{z}_{C}+\left(c_{3} d_{3}-c_{4} d_{4}\right) \dot{\varphi}-c_{3} \dot{z}_{3}-c_{4} \dot{z}_{4} \\
& +\left(4 k_{z}+k_{3}+k_{4}\right) z_{C}+\left(k_{3} d_{3}-k_{4} d_{4}\right) \varphi-k_{3} z_{3}-k_{4} z_{4}=F(t) \sin \beta_{0} \\
& J_{C} \ddot{\varphi}+\left(4 c_{x} a+c_{1} e_{1}-c_{2} e_{2}\right) \dot{x}_{C}+\left(c_{3} d_{3}-c_{4} d_{4}\right) \dot{z}_{C}-c_{1} e_{1} \dot{x}_{1}+c_{2} e_{2} \dot{x}_{2}-c_{3} d_{3} \dot{z}_{3}+c_{4} d_{4} \dot{z}_{4} \\
& +\left(4 c_{x} a^{2}+4 c_{z} b^{2}+c_{1} e_{1}^{2}+c_{2} e_{2}^{2}+c_{3} d_{3}^{2}+c_{4} d_{4}^{2}\right) \dot{\varphi}-k_{1} e_{1} x_{1}+k_{2} e_{2} x_{2}-k_{3} d_{3} z_{3}+k_{4} d_{4} z_{4} \\
& +\left(4 k_{x} a+k_{1} e_{1}-k_{2} e_{2}\right) x_{C}+\left(k_{3} d_{3}-k_{4} d_{4}\right) z_{C}+\left(4 k_{x} a^{2}+4 k_{z} b^{2}+k_{1} e_{1}^{2}+k_{2} e_{2}^{2}+k_{3} d_{3}^{2}+k_{4} d_{4}^{2}\right) \varphi=0 \\
& m_{1} \ddot{x}_{1}+c_{1} \dot{x}_{1}-c_{1} \dot{x}_{C}-c_{1} e_{1} \dot{\varphi}+k_{1} x_{1}-k_{1} x_{C}-k_{1} e_{1} \varphi=0 \\
& m_{2} \ddot{x}_{2}+c_{2} \dot{x}_{2}-c_{2} \dot{x}_{C}+c_{2} e_{2} \dot{\varphi}+k_{2} x_{2}-k_{2} x_{C}+k_{2} e_{2} \varphi=0 \\
& m_{3} \ddot{z}_{3}+c_{3} \dot{z}_{3}-c_{3} \dot{z}_{C}-c_{3} d_{3} \dot{\varphi}+k_{3} z_{3}-k_{3} z_{C}-k_{3} d_{3} \varphi=0 \\
& m_{4} \ddot{z}_{4}+c_{4} \dot{z}_{4}-c_{4} \dot{z}_{C}+c_{4} d_{4} \dot{\varphi}+k_{4} z_{4}-k_{4} z_{C}+k_{4} d_{4} \varphi=0
\end{aligned}
$$


In the above equations: $m_{i}, k_{i}$ and $c_{i}$ denote the mass, stiffness and damping coefficient of the $i$-th damper, respectively.

\section{Results of numerical calculations}

The press drive shaft operates at a constant angular velocity (except for acceleration and braking), so the use of the resonant devices is justified, although they are more sensitive to inaccurate tuning from devices with damping appliances. The possibility of effective vibration attenuation using the single horizontal absorber, the single vertical absorber and the system of two absorbers (one horizontal and one vertical) will be examined.

In the calculations it was assumed the harmonic excitation force: $F(t)=F_{0} \sin \omega t$, where $F_{0}=$ const . The system of differential equations (1)-(7) was solved using the prediction method. In order to determine the necessary coefficients (standing by functions $\sin (\omega t)$ and $\cos (\omega t))$ for each of the variables: $x_{C}, z_{C}, \varphi, x_{1}, x_{2}, z_{1}, z_{2}$, the linear system of algebraic equations with 14 unknowns had to be solved.

The aim of the calculations is to find the positions of dynamic vibration absorbers, tuned to the excitation force frequency, so that it is possible to eliminate or significantly reduce the forces transmitted to the floor by the machine supports.

As already mentioned, in practice the excitation force is mostly of the polyharmonic type. Determining the optimal positions of absorbers for each excitation frequency (in the band containing all natural frequencies of the press) will allow to find the optimal positions of absorbers designed to attenuate the selected harmonics of the excitation polyharmonic force.

The amplitudes of the horizontal reaction forces at the left and right side of the machine are always the same, while the amplitudes of the vertical reaction forces in the left and right supports do not have to be equal. Although the horizontal and vertical components of the forces in supports do not have to be in the same phase, an equivalent dimensionless reaction force is introduced in the following form:

$$
\tilde{R}_{e q}=\frac{1}{\left|F_{0}\right|} \sqrt{R_{x}^{2}+R_{z}^{2}}
$$

where: $R_{x}=R_{x L}=R_{x R}, R_{z}=\max \left\{R_{z L}, R_{z R}\right\}$.

In the above expressions the following notations are introduced: $R_{x L}, R_{x R}\left(R_{z L}, R_{z R}\right)-$ the amplitudes of the horizontal (vertical) components of the reaction force at the left and right side of the machine.

In the calculations the mass of the individual absorber (i.e. horizontal $-m_{1}$, vertical $m_{3}$ ) equal to $300 \mathrm{~kg}$ was assumed, about $2.7 \%$ of the mass of the press. The stiffness of the horizontal (vertical) absorber (tuned to the excitation force frequency) is given by: $k_{1}=m_{1} \omega^{2} \quad\left(k_{3}=m_{3} \omega^{2}\right)$.

For the case of only one absorber attached, it was assumed as the objective function the equivalent dimensionless reaction force dependent on the parameter $e_{1}\left(d_{3}\right)$ describing position of the horizontal (vertical) absorber: $\tilde{R}_{e q}=\tilde{R}_{e q}\left(e_{1}\right)\left(\tilde{R}_{e q}=\tilde{R}_{e q}\left(d_{3}\right)\right)$. The optimal positions of the absorbers were determined using the gradient method, searching for the minimum value of the objective function the following limitations were imposed: $\left|e_{1}\right| \leq 0.8 \mathrm{~m},\left|d_{3}\right| \leq 0.9 \mathrm{~m}$. 
The amplitudes of the dimensional forces transmitted in the horizontal and vertical directions to the supports, on the left $\left(R_{x L} /\left|F_{0}\right|\right)$ and right side $\left(R_{z L} /\left|F_{0}\right|, R_{z R} /\left|F_{0}\right|\right)$, for the press without the absorbers attached, are shown in Fig. 4. The diagrams in Fig. 4 present also the equivalent dimensionless reaction forces for the press without the absorbers, and for the press with the single absorber, horizontal or vertical, attached at the optimal positions.

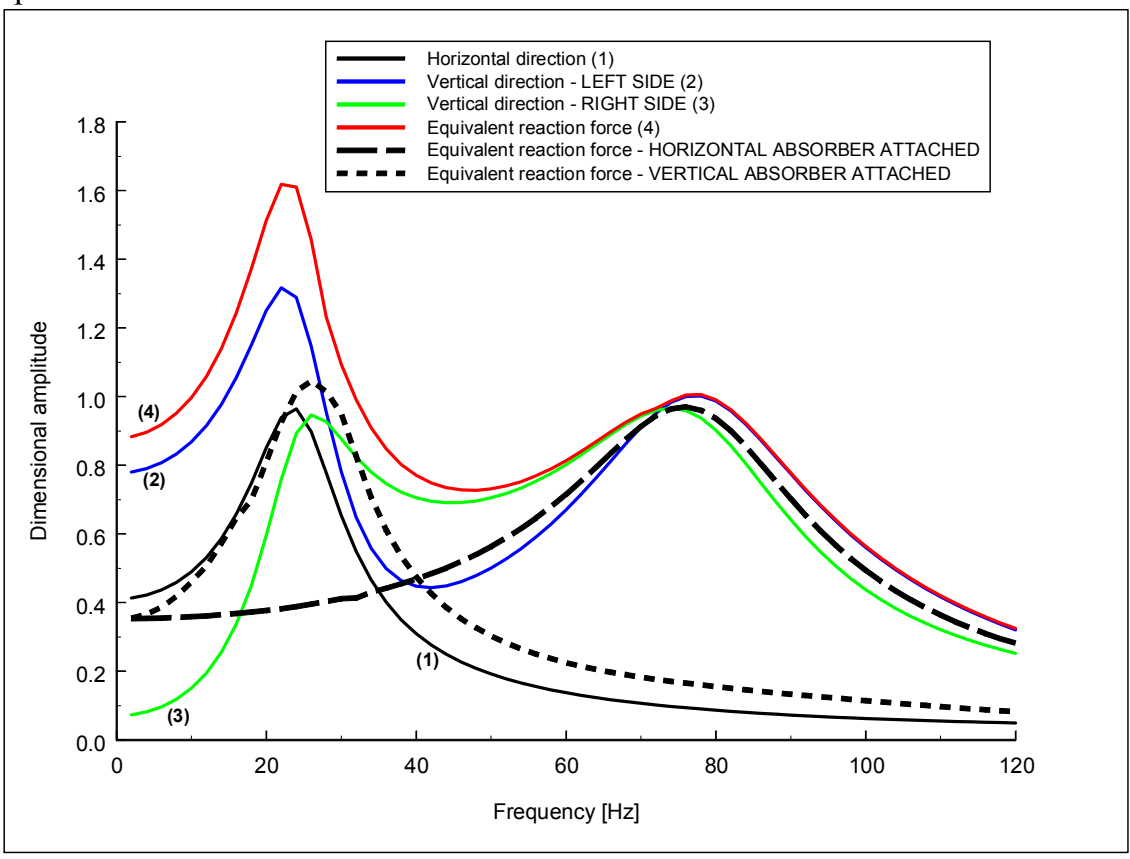

Fig. 4. Dimensionless amplitudes of the transmitted forces for the press without and with the single absorbers attached.

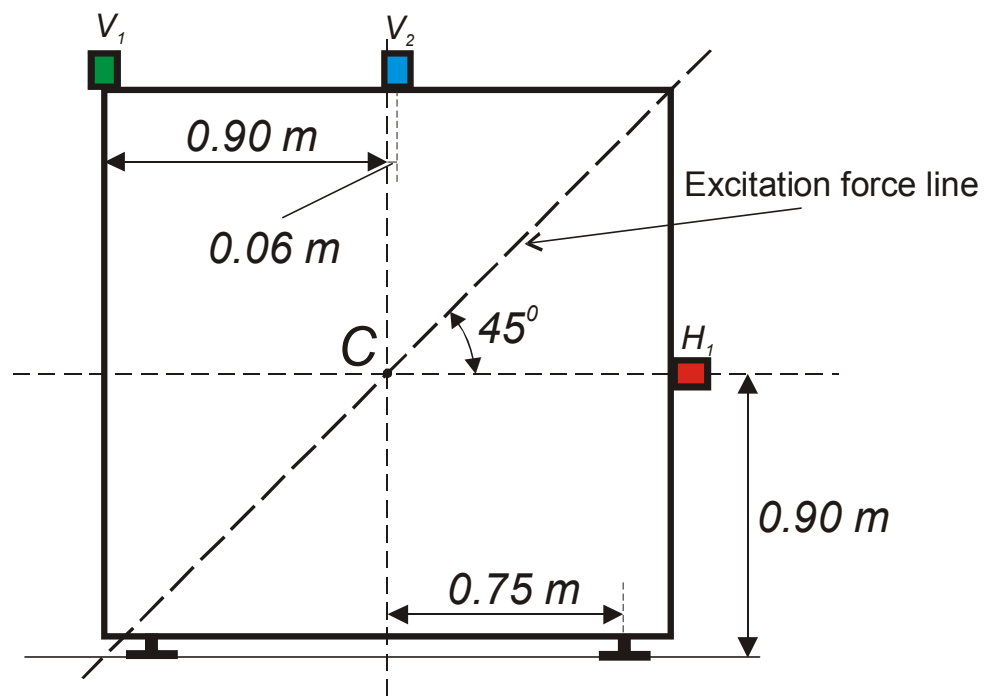

Fig. 5. Optimal positions of the single horizontal $\left(H_{l}\right)$ and single vertical $\left(V_{l}, V_{2}\right)$ absorber: $H_{l}$ - the same position for each frequency; $V_{l}-$ for: $0<f<30 \mathrm{~Hz} ; V_{2}-$ for: $f>42 \mathrm{~Hz}$. 
It is evident that the properly placed horizontal absorber is more effective up to a frequency of around $40 \mathrm{~Hz}$, above this frequency the properly located vertical absorber is more effective. It turns out that the optimal position of the horizontal absorber is always the same - at the height of the machine's mass center: $e_{1}=0.0 \mathrm{~m}$ (Fig. 3, Fig. 5). The optimal position of the vertical absorber depends on the frequency band: up to $30 \mathrm{~Hz}$ it is the extreme admissible left position: $d_{3}=-0.9 \mathrm{~m}$; for the frequencies between $30 \mathrm{~Hz}$ and $42 \mathrm{~Hz}$ the optimal position lies in the range $0.04 \mathrm{~m}<d_{3}<0.06 \mathrm{~m}$; for the frequencies above $42 \mathrm{~Hz}$ this is the place described by the value: $d_{3}=0.07 \mathrm{~m}$ (Fig. 3, Fig. 5).

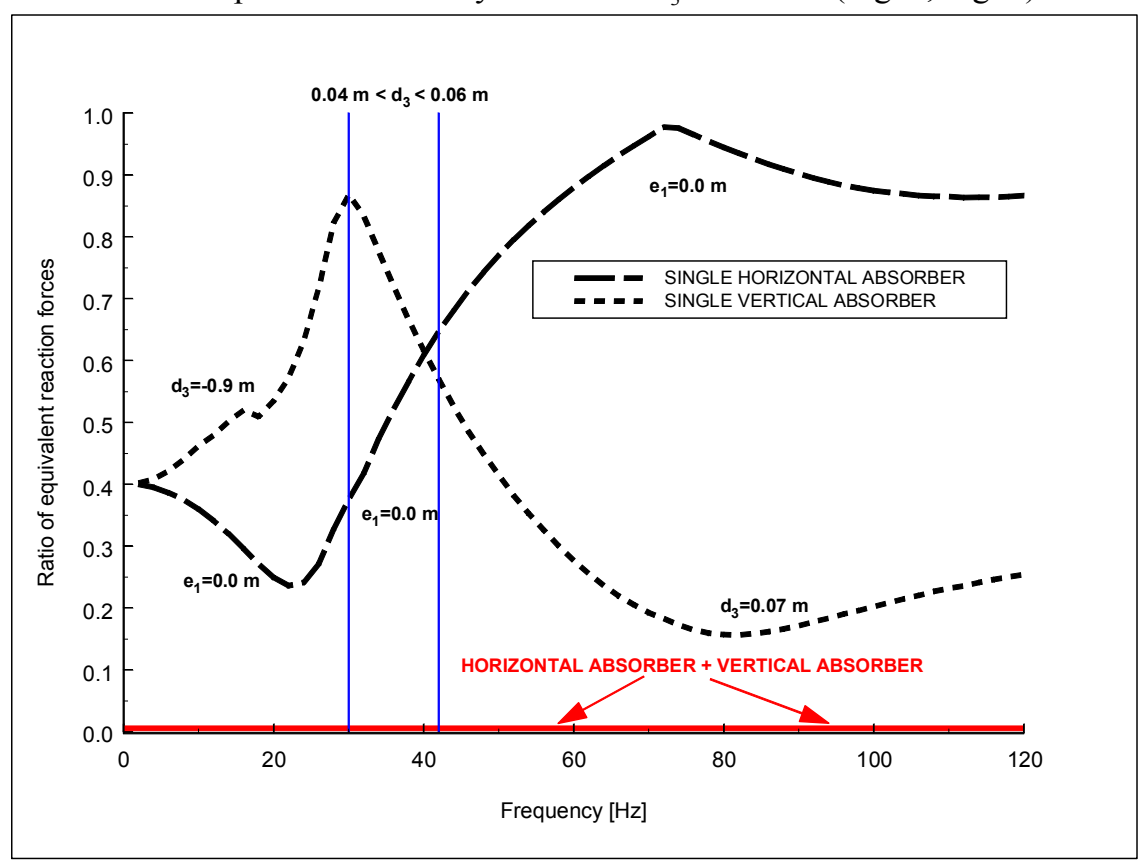

Fig. 6. Ratio of the equivalent reaction forces for the system with absorbers to its value for the machine without the absorbers attached.

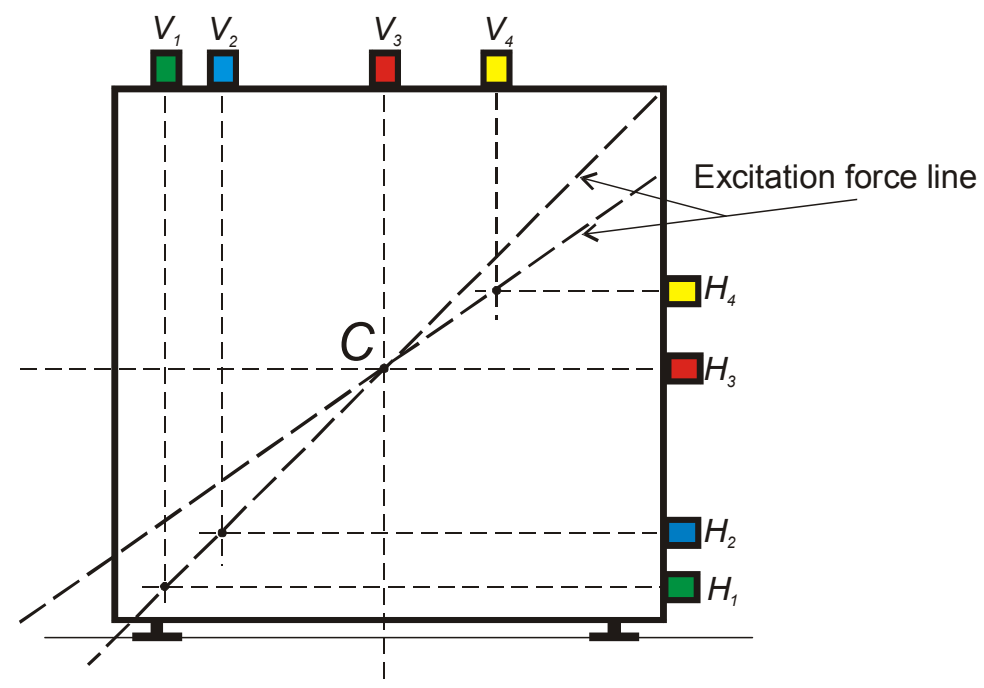

Fig. 7. Exemplary optimal positions of components of the double-absorber system $\left[H_{i}, V_{i}\right]$. 
In order to better investigate effectiveness of the absorbers, in Fig. 6 the ratio of the equivalent reaction force for the system with absorbers to its value for the machine without the absorbers is shown. It is visible that the vibrations suppression efficiency of the single absorbers is limited, especially in the range between $35 \mathrm{~Hz}$ and $45 \mathrm{~Hz}$. The highest effectiveness of the horizontal absorber is achieved around the first natural frequency of the press $\left(f_{1}=24 \mathrm{~Hz}\right)$, while for the vertical absorber around the second natural frequency ( $f_{2}=78 \mathrm{~Hz}$ ) which is the frequency of the vertical, uncoupled vibration mode.

The results of calculations showed that application of the system of two properly located absorbers (one horizontal and one vertical) allows to completely eliminate the vibrations of the machine. The optimal positions are such that the absorber motion lines should cross at the same point the action line of the excitation force (this ensures that the sum of moments of all forces with respect to this point is equal to zero). This conclusion is true regardless of the angle of inclination of the force line (for $0^{\circ}<\beta_{0}<90^{\circ}$ ) and valid independent of the mass of the absorbers. The examples of the optimal double-absorber configurations are shown schematically in Fig. 7. For each pair of absorbers the reaction forces in the press supports are equal to zero. The choice of the best configuration may depend on additional conditions, because some positions may be excluded due to the technical (e.g. no assembly possible) or safety reasons.

\section{Conclusions}

In the paper the problem of the optimal distribution of dynamic vibration absorbers for the mechanical press subjected to harmonic excitation is presented. The obtained results allow to determine the optimal position of the single absorber, horizontal or vertical, and the optimal positions of the double-absorber system components, tuned to the excitation force frequency. The suppression efficiency of the single absorbers is limited, while the use of the double-absorber system, appropriately mounted, allows to completely eliminate the vibrations of the machine. The results obtained allow in practice to find the optimal positions of the absorbers tuned to the selected frequencies of harmonics of the polyharmonic excitation. It should be remembered that some location of absorbers may be limited by the technological or safety issues.

\section{References}

1. M. Abdel-Rohman, J.J. Mariam, J. Vib. Control 12(9), 1011-1046 (2006)

2. S.R. Chen, J. Wu, Eng. Struct. 30, 3316-3324 (2008)

3. F. Ricciardelli, J. Wind. Eng. Ind. Aerod. 89, 1539-1551 (2001)

4. J. Snamina, P. Martynowicz, W. Łatas, Solid State Phenomen. 208, 29-39 (2014)

5. M. Luu, V. Zabel, C. Könke, Finite Elem. Anal. Des. 53, 13-23 (2012)

6. Quan. Li, J. Fan, J. Nie, Quanwang. Li, Y. Chen, J. Sound Vib. 329, 4068-4092 (2010)

7. Y. Nakano, H. Takahara, E. Kondo, J. Sound Vib. 332, 1626-1638 (2013)

8. F. Rüdinger, Eng. Struct. 28, 1774-1779 (2006)

9. S. Sgobba, G.C. Marano, Mech. Syst. Signal Pr. 24, 1739-1755 (2010)

10. O.F. Tigli, J. Sound Vib. 331, 3035-3049 (2011)

11. W. Łatas, J. Theor. App. Mech.-Pol. 53(2), 467-476 (2015)

12. C. Min-Li, Chi-C. Lin, Jin-M. Ueng, Kai-H. Hsieh, Jer-F. Wang, Struct. Control Health Monit. 17, 532-548 (2010) 